\title{
Management of the Patient with Heart Failure and an Implantable Pulmonary Artery Hemodynamic Sensor
}

\author{
Jacob Abraham ${ }^{1} \cdot$ Patrick J. McCann ${ }^{2}$ - Maya E. Guglin ${ }^{3} \cdot$ Arvind Bhimaraj $^{4} \cdot$ Terrie-Ann S. Benjamin $^{5}$. \\ Monique R. Robinson ${ }^{6}$. Orvar T. Jonsson ${ }^{7}$. Scott C. Feitell ${ }^{8}$. Kunjan A. Bhatt ${ }^{9} \cdot$ Mosi K. Bennett $^{10}$ • J.T. Heywood ${ }^{11} \cdot$ on \\ behalf of Hemodynamic Frontiers in Heart Failure (HF2) Investigators
}

Published online: 14 July 2020

(C) The Author(s) 2020

\begin{abstract}
Purpose of Review Heart failure (HF) management guided by hemodynamics obtained from an implantable pulmonary artery pressure (PAP) sensor (CardioMEMS) improves symptoms and reduces HF hospitalizations (HFH). This paper reviews the theoretical basis of pulmonary vascular physiology, summarizes recently published data about CardioMEMS, and provides practical guidelines for patient selection and management.

Recent Findings Compared to patients managed by standard care, HF patients randomized to PAP-guided treatment have a higher frequency of medication adjustments, resulting in lower PAP and fewer HFH. Real-world analyses further support associations between implant of the CardioMEMS sensor with reductions in PAP, hospitalizations, and mortality.

Summary Implantable, wireless hemodynamic sensor technology is a promising remote monitoring platform for chronic HF. A phased approach using a treatment algorithm may improve the efficiency and effectiveness of pressure-guided therapy.
\end{abstract}

Keywords Pulmonary artery $\cdot$ Pulmonary hypertension $\cdot$ Heart failure $\cdot$ Hemodynamics $\cdot$ Remote monitoring

\section{Abbreviations}

ACE-I Angiotensin-converting-enzyme inhibitor

ARB Angiotensin receptor blocker

APP Advanced practice provider

BMI Body mass index

BPM Beats per minute
CHAMPION CardioMEMS Heart Sensor Allows
Monitoring of Pressure to Improve Outcomes in NYHA Class III Heart Failure Patients
CI
$\mathrm{CpcPH}$
Confidence interval
Combined pre-capillary and post-capillary pulmonary hypertension

This article is part of the Topical Collection on Technology and Cardiovascular Health

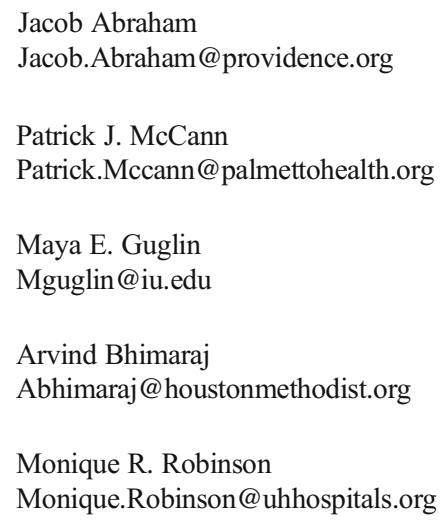

\author{
Orvar T. Jonsson \\ Orvar.Jonsson@sanford.health.org \\ Scott C. Feitell \\ Scott.Feitell@ rochesterregional.org \\ Kunjan A. Bhatt \\ Kunjan.Bhatt@hcahealthcare.com \\ Mosi K. Bennett \\ Mosi.Bennett@aline.com \\ J.T. Heywood \\ Heywood.James@scrippshealth.org
}

Extended author information available on the last page of the article 


\begin{tabular}{|c|c|}
\hline DPG & Diastolic pressure gradient \\
\hline DSRC & $\begin{array}{l}\text { Device-related or system-related } \\
\text { complications }\end{array}$ \\
\hline $\mathrm{EF}$ & Ejection fraction \\
\hline GFR & Glomerular filtration rate \\
\hline GUIDE-HF & $\begin{array}{l}\text { Hemodynamic-GUIDEd Management of } \\
\text { Heart Failure }\end{array}$ \\
\hline $\mathrm{HF}$ & Heart failure \\
\hline HFH & Heart failure hospitalization \\
\hline HFpEF & Heart failure with preserved ejection fraction \\
\hline HFrEF & Heart failure with reduced ejection fraction \\
\hline HR & Hazard ratio \\
\hline IV & Intravenous \\
\hline LA & Left atrium \\
\hline LVAD & Left ventricular assist device \\
\hline NT-proBNP & N-terminal pro-brain natriuretic peptide \\
\hline NYHA & New York Heart Association \\
\hline PA & Pulmonary artery \\
\hline PAP & Pulmonary artery pressure \\
\hline PADP & Pulmonary artery diastolic pressure \\
\hline PCWP & Pulmonary capillary wedge pressure \\
\hline PVR & Pulmonary vascular resistance \\
\hline $\mathrm{PH}$ & Pulmonary hypertension \\
\hline PVR & Pulmonary vascular resistance \\
\hline RV & Right ventricle \\
\hline SGLT2 & Sodium-glucose transport protein 2 \\
\hline WU & Woods' units \\
\hline
\end{tabular}

\section{Introduction}

The final common pathway of congestion in heart failure (HF), regardless of ejection fraction (EF), is related to increases in intra-cardiac pressures. Ambulatory hemodynamic monitoring allows for detection of these pressure changes prior to the onset of symptoms, enabling earlier interventions to lower pressures, avoids hospitalization, and improves quality of life. [1] By avoiding decompensation, pressure-guided HF management may also delay HF progression and improve survival. [2] By contrast, monitoring of symptoms or noninvasive surrogates of intra-cardiac pressure has not been consistently effective [3-6].

An ambulatory hemodynamic pulmonary artery pressure (PAP) sensor (CardioMEMS HF System, Abbott, Sylmar, $\mathrm{CA}$ ) has been approved to reduce HF hospitalization (HFH) in New York Heart Association (NYHA) class III patients with a HFH in the preceding 12 months. Utilizing microelectromechanical technology and powered by radiofrequency energy, the sensor is implanted into PA branches through a catheter-delivery system. Patients take daily home readings using an external electronics system that transmits the PAP data wirelessly to a secure website for clinician review. In a pivotal randomized trial, HF patients managed with the CardioMEMS system had lower PAP and fewer HFH than a control group with implanted monitor but without disclosure of pressures to clinicians. [1]

While the concept of hemodynamically tailored therapy has a long history, the recent availability of ambulatory pressure monitoring has refocused interest in hemodynamic management for earlier stages of disease and in an outpatient setting. [7] The ability to monitor hemodynamics longitudinally creates both opportunities and challenges. Foremost, guidelines for patient selection, monitoring, and management of ambulatory hemodynamics are lacking. As the field of ambulatory hemodynamic monitoring is expanding, there is a need for best practice recommendations to monitor and manage the patient with an implanted sensor. In this paper, we provide consensus recommendations from HF specialists experienced with hemodynamic-guided management of HF.

\section{Theoretical Considerations}

The pulmonary circulation is a low-pressure, highcapacitance vascular bed that accommodates large changes in flow with little or no increase in pressure. The pulmonary circuit is coupled to the right ventricle (RV), which is morphologically adapted to pump at low pressure. The RV:PA unit is in series with the left atrium (LA) and therefore LA pressure is a key determinant of PAP. Pulmonary artery diastolic pressure (PADP) is an adequate surrogate for pulmonary capillary wedge pressure (PCWP) and LA pressure in the absence of intrinsic pulmonary vascular pathology [8].

Pulmonary hypertension in the setting of left heart disease, classified as group 2 according to the World Health Organization classification, is common in $\mathrm{HF}$ and is associated with a worse prognosis than HF without $\mathrm{PH}[9,10]$. Group $2 \mathrm{PH}$ is defined hemodynamically as PCWP $>15 \mathrm{mmHg}$ with mean PAP $>=25 \mathrm{mmHg}$. In HF, LA hypertension increases the PA pressure and thereby RV afterload. Afterload of the $\mathrm{RV}$ is traditionally measured by parameters of precapillary disease (pulmonary vascular resistance (PVR), transpulmonary gradient, and diastolic pressure gradient (DPG)), whereas parameters that also incorporate pulsatile load (pulmonary effective elastance, pulmonary capacitance) are more predictive of RV dysfunction and prognosis [11]. Reduced compliance of the left atrium can also cause prominent $\mathrm{V}$-waves, increasing systolic load on the RV [12]. In some patients, the chronic increase in LA pressure causes pulmonary vascular remodeling resulting in a component of pre-capillary $\mathrm{PH}[\mathrm{PCWP}>=15$, PVR $>$ $3 \mathrm{WU}$, and increased DPG (PAD-PCWP) >3]. These individuals with combined post- and precapillary $\mathrm{PH}$ $(\mathrm{CpcPH})$ have worse prognosis compared with isolated postcapillary pulmonary hypertension [13]. 
With persistently increased total afterload, the RV hypertrophies, dilates, and eventually develops overt contractile dysfunction and worsening tricuspid regurgitation. The development of RV dysfunction is an ominous development associated with markedly worse outcomes in HF [14].

\section{Evidence Review}

In the CardioMEMS Heart Sensor Allows Monitoring of Pressure to Improve Outcomes in NYHA Class III Heart Failure Patients (CHAMPION) trial, 550 patients with NYHA class III HF and a HFH in the prior 12 months underwent right heart catheterization and implant of a CardioMEMS sensor. Patients were then randomized to management guided by pressure data versus traditional care directed by signs and symptoms. The primary endpoint of HFH at 6 months was reduced by $28 \%$ (HR 0.72 , CI $0.60-0.85, p=$ 0.002 ). Outcomes were similarly improved in patients with reduced and preserved systolic function. The reduction in $\mathrm{HFH}$ was associated with reduction in PAP, and increased frequency of medication changes. [1] Diuretics and vasodilators were adjusted nearly three times more commonly than in the treatment group. [15] Despite more intensive diuretic management, there were no significant changes in renal function, including in those with chronic kidney disease.

The primary safety endpoints of device-related or systemrelated complications (DSRC) were also achieved (98.6\% freedom from DSRC) with no sensor failures. In 575 implant attempts, there were 15 serious adverse events: eight (1\%) were DSRC and 7 were procedure-related complications.

Several studies of the real-world use of CardioMEMS have been published. Heywood et al. reported outcomes of the first 2000 patients managed with the CardioMEMS system following FDA-approval $[16 \bullet \cdot]$. Compared with the CHAMPION population, post-approval patients were older with a greater proportion of females and HF with preserved $\mathrm{EF}$ (HFpEF). These patients were adherent with daily transmissions and demonstrated greater reductions in PA pressure that were achieved in CHAMPION. Desai et al. used Medicare claims data to compare rates of HFH in 1114 patients pre- and postimplantation [17••]. Sensor implant was associated with a $51 \%$ reduction in cumulative $\mathrm{HFH}$ at 6 months that was sustained at 12 months. In a separate analysis, a propensity matched cohort study of Medicare beneficiaries showed an association of implant of a CardioMEMS sensor with reduced HF hospitalization ( 0.65 vs 0.88 ; HR, 0.76 [95\% CI, 0.650.89 ]; $p<0.001)$ as well as all-cause mortality $(0.23$ deaths per year vs 0.30 deaths per year; HR, 0.70 [95\% CI, 0.590.83]; $P<0.001)$ [18].

Vaduganathan et al. [19] studied post-approval adverse events reported in the Manufacturer and User Facility Device Experience (MAUDE) database. Of more than 5500 total implants, there were 28 reports of PA injury/hemoptysis $(0.5 \%)$ and 22 total deaths $(0.4 \%)$ (6 after PA injury/hemoptysis, 4 HF-related, and 12 unknown or likely unrelated). Sensor failure, malfunction, or migration was reported in 46 cases $(0.8 \%)$.

Relatively little is published about specific management approaches based on sensor pressure data. In CHAMPION, specific medical interventions were left to the discretion of investigators. A retrospective study from an advanced HF center showed that a nurse-supervised treatment algorithm is feasible and is associated with significant improvements in $\mathrm{PAP}$ and reduction in $\mathrm{HFH}\left[20^{\circ}\right]$.

\section{Patient Selection and Education}

Clinical and social factors should aid in refining selection of patients that meet the approved indication for CardioMEMS (see Table 1). Prior to device implant, the patient and caregiver(s) should be instructed on the use of the home electronics unit and the data transmission process to promote adherence with data transmission and to reduce the likelihood of user error.

\section{Initial Hemodynamics and Device Calibration}

During the right heart catheterization, careful measurement of PCWP, PAP, cardiac output, and vascular resistances should be made. As PADP is the main parameter of interest during management, accurate measurement is critical. If there is significant respiratory variation, a brief unforced breath hold can assist measurement of end-expiratory PADP. Additional care is needed to measure the PCWP and the difference between PADP and PCWP (e.g., DPG). A DPG $\geq 5 \mathrm{mmHg}$ may indicate concomitant pre-capillary pulmonary hypertension [21]. If the systemic vascular resistance and PCWP are both elevated, administration of a short-acting vasodilator such as

\section{Table 1 Considerations for CardioMEMS patient selection}

May not be appropriate for:

- BMI $>35$ and chest circumference $>165 \mathrm{~cm}$

- Complex congenital heart disease or mechanical right-sided heart valve replacement

- Hypersensitivity to aspirin and/or clopidogrel

- Recurrent $(>1)$ deep venous thrombosis or pulmonary embolism

- Patients with advanced kidney disease with GFR $<25 \mathrm{ml} / \mathrm{min} / 1.73 \mathrm{~m}^{2}$ who are non-responsive to diuretic therapy or who are on chronic dialysis

Additional considerations:

- Ability to adhere to medical therapy

- Access to pharmacy and medications

- Ability to communicate frequently with clinician 
nitroprusside can help assess the relative contribution of elevated vascular resistance to the elevation in filling pressures. A significant reduction in filling pressures during such a vasodilator challenge may support preferential use of vasodilators during ambulatory management and can unmask significant PADP-PCWP gradients.

\section{Principles of Management}

In our experience, the key steps in ambulatory hemodynamic monitoring are establishing a target PADP and pro-actively treating pressure values outside of the goal range. A treatment algorithm can be effective for managing individual patients and supports operational efficiency with large numbers of patients. One suggested treatment approach, adapted by the present authors from a single center experience, is adapted in Fig. 1 [21].

HF management targeting pulmonary artery pressures should be approached in 3 phases:

- Immediate post-implant period (0-7 days) when PADP baseline ranges are established;

- Optimization phase when intense medication management and close monitoring are applied to PADP goal;

- Maintenance period when additional adjustment of neurohormonal drugs, vasodilators, and diuretics is added or titrated as needed to maintain or improve pressures, and/ or the PADP goal is re-assessed.

\section{Phase I 0-7 Days}

Within the first week after implant, the patient's transmitted pressure data should be reviewed. The clinician should then define a PADP goal based upon the baseline PAP, taking into account individual patient factors such as ejection fraction, systemic blood pressure, and renal function. A range of PADP, referred to as the threshold range, is programmed into
Merlin.net to trigger out of range notifications. In the early post-implant phase, this threshold range should be broad (e.g., $10 \mathrm{mmHg}$ ), with the PADP target centered within the specified range. For example, if the target PADP is $15 \mathrm{mmHg}$, the threshold range would be programmed at $10-20 \mathrm{mmHg}$.

\section{Phase II-Optimization}

The key objective during this phase is to establish the optimal achievable PADP goal, which may take up to 90 days post implant to determine. Initially, a PADP target of $<20 \mathrm{mmHg}$ can be set for most patients. Higher PADP targets may be required for patients observed to have PADP-PCWP > $5 \mathrm{mmHg}$. Pressure data is reviewed 2-3 times per week with medications adjusted to achieve the PAD goal (Fig. 1). As discussed below, diuretics are the most frequently adjusted medication, though vasodilators should be considered in those with elevated systemic vascular resistance.

The magnitude and rate of pressure deviation should be considered in the decision to treat. For example, providers may intervene after:

- A 3-5 mmHg change in PADP over 2-3 days

- A $5 \mathrm{mmHg}$ or more change in PADP in 1 day.

Sudden changes in pressure can be artifactual, and therefore review of the pressure waveform is recommended if pressure readings are suspect.

As noted earlier, the ultimate PADP goal for a patient must be individualized based on renal function, systemic blood pressure, and the presence of symptoms of hypoperfusion. During active pressure management, renal function and electrolytes should be measured within a week of each medication adjustment to detect acute renal dysfunction or electrolyte derangements. Some may not be able to attain the prespecified PADP goal without significant deterioration of renal function or hypotension. In these cases, the PADP goal should be increased as need to maintain clinical stability. Conversely, once
Fig. 1 Phases and goals of hemodynamic monitoring. Suggested phased approach to hemodynamic management with iterative medication adjustment based on HF phenotype and PAD target

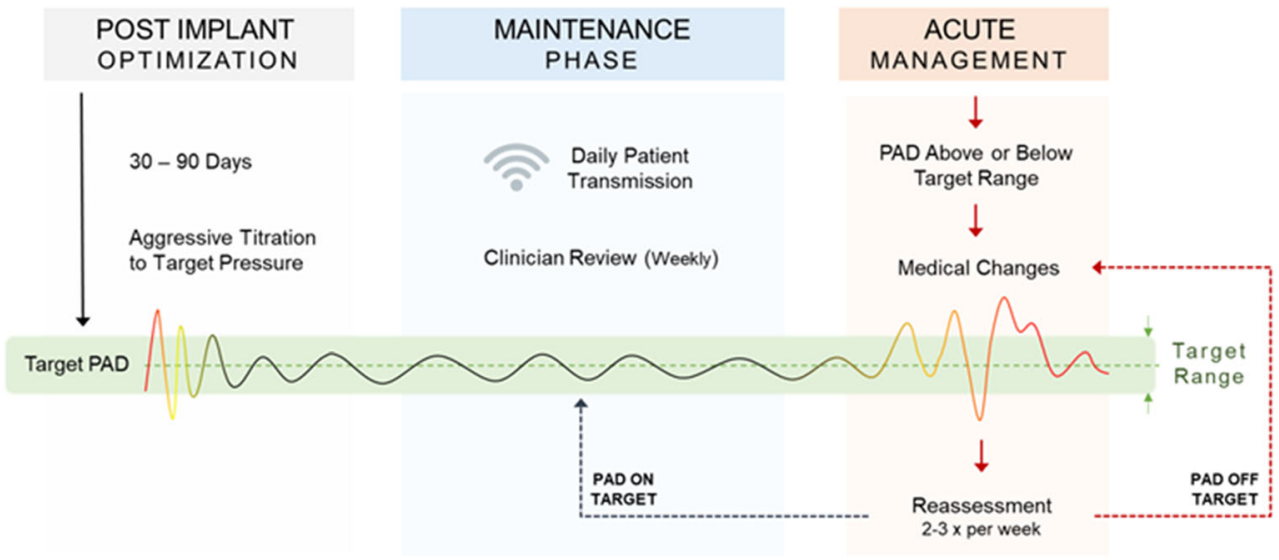


the initial PADP goal is achieved, a new lower goal may be selected to optimize the patient.

\section{Phase III-Maintenance Phase}

Once the optimal achievable PADP has been determined, the PADP threshold range should be narrowed to $\sim 5 \mathrm{~mm} \mathrm{H}$ (e.g., 2-3 mmHg above and below the target PADP). The managing provider(s) can rely on email notifications from the Merlin.net system to identify out-of-range patients. Individual patient data can be reviewed only once per month, with reassessment of medications and threshold ranges as needed. Management during this time is simplified in that clinicians are only alerted if pressures are outside of these ranges for 3 consecutive readings. Although pressures may have normalized, clinicians should continue to review patient medications and device therapy to ensure the optimal guideline directed care is being provided.

\section{Heart Failure with Reduced Ejection Fraction (HFrEF)}

HFrEF patient should be treated optimally with guideline-directed medical therapy and indicated implantable devices prior to considering a wireless hemodynamic sensor. Despite appropriate management, a significant portion of patients experience NYHA class III symptoms and require hospitalization for acute decompensation episodes.

\section{Diuretics}

Loop diuretics are an essential component of therapy for most HF patients to address the congestive clinical signs and symptoms caused by fluid and sodium retention. Because these diuretics require active secretion into the tubular lumen, a threshold dose must be administered to produce to diuretic an effect, with a sigmoid-shaped relationship between urinary concentration and fractional sodium excretion. Diuretic resistance is common and is caused by excessive sodium intake, post-diuretic sodium retention, and diminished natriuretic effect from chronic diuretic use $[22,23]$. In comparison with furosemide, bumetanide and torsemide have the advantage of greater and more consistent bioavailability following oral dosing $[24,25]$.

A PADP greater than $5 \mathrm{mmHg}$ should prompt an increase in the dose of loop diuretics or volume-altering agents such as sacubritril/valsartan (Fig. 2). Frequent (every 3-7 days) incremental dose adjustments (25-50\% in furosemide equivalents) rather than larger, infrequent doses may cause less renal dysfunction and electrolyte disturbances. If hemodynamic congestion persists despite the increase in diuretic dose, options include switching to a different loop diuretic, combination diuretic therapy with sequential nephron blockade, and outpatient IV diuretic administration. In a large, propensity-adjusted analyses, metolazone use was associated with increased renal insufficiency, hyponatremia, hypokalemia, and mortality compared to loop diuretic use in hospitalized HF patients. [26] Increasing doses of loop diuretics may therefore be a preferable strategy unless diuretic resistance persists.

In patients with significant congestion, e.g., $>10 \mathrm{mmHg}$ above goal PADP, more aggressive treatment is appropriate. Diuretics can be increased $50-100 \%$ above baseline until pressures have returned to within $5-10 \mathrm{mmHg}$ of the goal. Alternatively, IV diuretics can be provided in an outpatient setting or thiazide diuretics added for a short time with close laboratory monitoring. [27]

Serum creatinine may increase during decongestive therapy for acute heart failure. Worsening renal function is associated with low systolic blood pressure, venous congestion, elevated intra-abdominal pressure, and neurohormonal activation, rather than low cardiac output [28, 29]. While significant deterioration in renal function should be avoided, a small decline in renal function is not associated with renal tubular injury in the context of diuresis [30]. Testani et al. have shown that improvement in hemodynamics coupled with a small decline in estimated GFR actually carries an improved prognosis [31].

\section{Modification of Neurohormonal Therapy}

The benefits of neurohormonal drugs are well established in HFrEF. Hemodynamic optimization may provide incremental benefit to neurohormonal blockade in HFrEF. Givertz et al. showed that HFrEF patients in CHAMPION receiving both ACE-I and beta blocker had a $43 \%$ lower HFH and 57\% lower mortality $(p=0.06)$ than controls in CHAMPION [2]. Following implant of a hemodynamic sensor, continued attempts to achieve target doses of neurohormonal drugs including sacubritril/valsartan should be made, as patients previously intolerant of evidence-based HF therapies may respond favorably following improvement of hemodynamics [32].

Sacubitril/valsartan reduces the risk of death and $\mathrm{HFH}$ in HFrEF patients with NYHA class II or III symptoms who tolerate an ACE-I or ARB [33]. Replacing ACE-I or ARB with sacubitril/valsartan is therefore recommended for symptomatic patients. Owing to the natriuetic and diuretic effects of neprilysin inhibition, loop diuretic doses may need to be decreased when starting/titrating sacubitril/valsartan. The CardioMEMS sensor may aid in maximizing the dose sacubitril/valsartan through optimizing filling pressures.

\section{Heart Rate}

In addition to PA pressures, implantable hemodynamic monitors provide snapshots of heart rate and rhythm. This data should be used in conjunction with PA pressures data to optimize beta blockade. As HR is lowered, there is decreased load on the right 
a

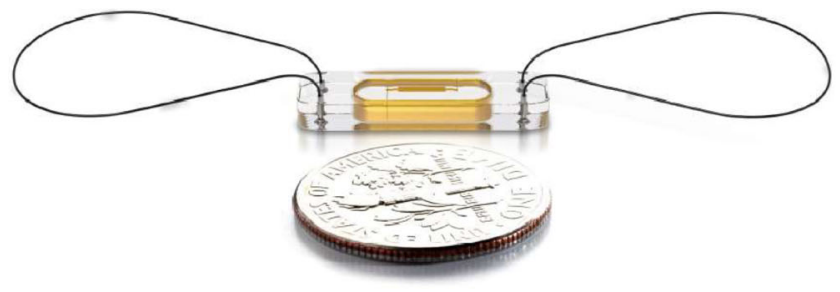

b

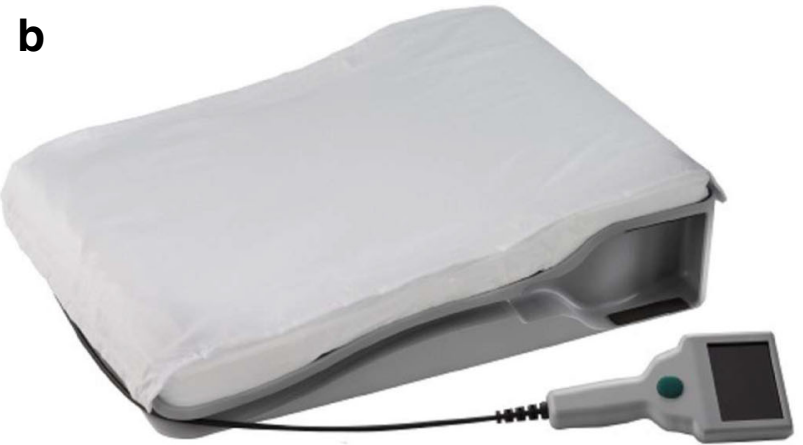

C

From: 01-10-2020 To: 04-09-2020

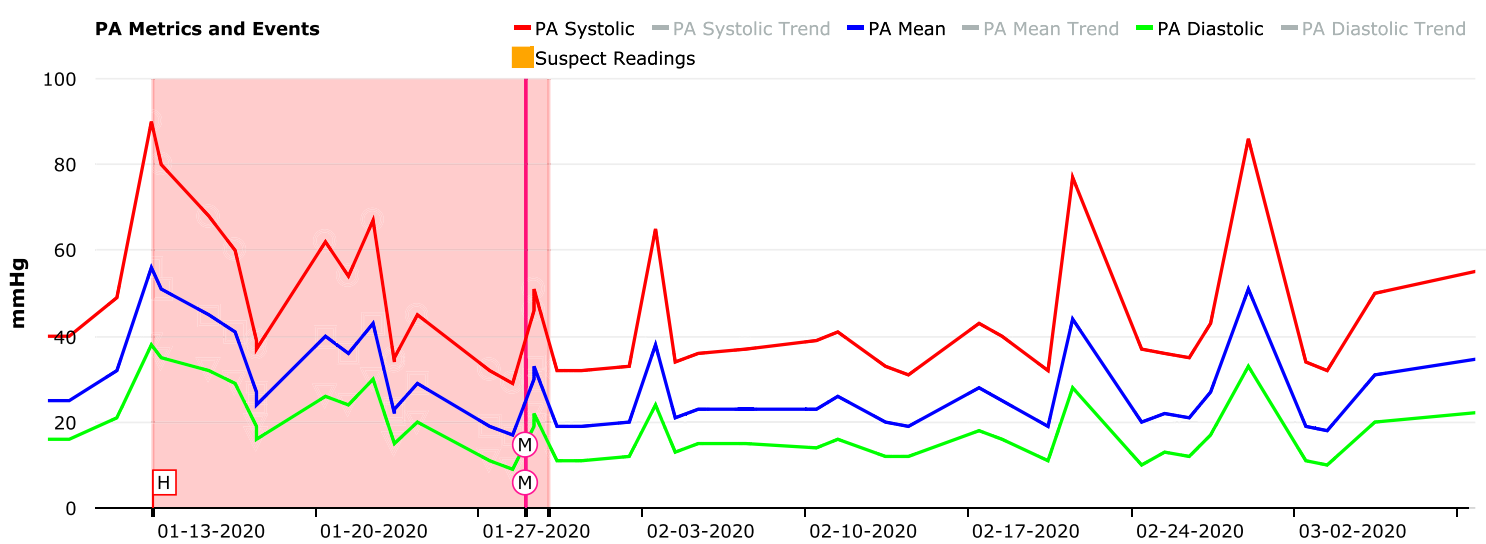

Fig. 2 CardioMEMS device and pressure trends. a CardioMEMS sensor (reprinted with permission from Abbott). b Patient home electronics units (reprinted with permission from Abbott). c Trend of pulmonary artery

ventricle [34]. In HFrEF patients in sinus rhythm with heart rate $>70 \mathrm{bpm}$ despite maximally tolerated beta blocker, ivabradine may be considered to reduce heart failure hospitalizations [35].

\section{Heart Failure with Preserved Ejection Fraction}

Despite intense study over the last decade, there are no proven pharmacologic therapies for HFpEF. Management is focused on the control of blood pressure, relief of congestion, treatment of CAD per contemporary guidelines, maintenance of sinus rhythm, and treatment of comorbid conditions [36]. Due to the complex pathophysiology in HFPEF, small changes in intravascular volume produce marked changes in intra-cardiac pressures [37]. Consequently, the ability to monitor PA pressure and heart rate/rhythm makes implantable hemodynamic monitoring an appealing strategy in the management of HFpEF patients. In fact, a prespecified analysis from the CHAMPION trial demonstrated PA pressure monitoring produced an even greater benefit in patients with $\mathrm{HFpEF}$ versus HFrEF [38].

Given the increased slope of the pressure-volume relationship in HFpEF, a narrow PADP threshold range is appropriate. Diuretic doses should be adjusted by $25-50 \%$ to address pressure deviations from the goal. In patients with GFR > systolic (red), diastolic (green), and mean (blue) pressures in HFpEF patient. Vertical lines indicate temporary medication changes ("T") to address pressure changes. Red-shaded area indicates hospitalization

$30 \mathrm{ml} / \mathrm{m}^{2} / 1.73 \mathrm{~m}^{2}$ and serum potassium $<5.0 \mathrm{mEq} / \mathrm{L}$, aldosterone receptor antagonists can be used in high-doses (e.g., spironolactone $75-300 \mathrm{mg}$ daily) to promote diuresis while maintaining serum potassium levels [39]. A randomized control trial of high-dose spironolactone in acute HF, however, showed no benefit of spironolactone $100 \mathrm{mg}$ daily on natriuretic peptide levels or markers of congestion at $96 \mathrm{~h}$ [40]. While another randomized study of spironolactone in HFpEF showed no benefit on the combined end-point of cardiovascular death, aborted cardiac arrest, or hospitalization for HF, the spironolactone group had a lower incidence of HF hospitalization [41]. Professional guidelines consequently provide a class $\mathrm{IIb}$ recommendation for the use of aldosterone receptor antagonists to reduce hospitalizations [42].

Vasodilators have a secondary role in the management of HFpEF. In the prespecified sub-group analysis of HFpEF patients in CHAMPION, only $12 \%$ of medication changes in the treatment arm were changes in vasodilator therapy [38]. Additionally, a randomized, double-blind, placebocontrolled cross-over study showed that HFpEF patients who received isosorbide mononitrate were less active (as measured by wearable accelerometer) in a dose-dependent fashion 
and had similar 6-min walk distance and quality of life scores as patients who received placebo [43].

\section{Program Structure}

Clinic personnel should be assigned to monitor pressure data, receive email notifications, and communicate treatment recommendations to the patient. A nurse or advanced practice provider (APP) is well-suited for this position and can incorporate monitoring with other clinical responsibilities so that new personnel rarely need to be hired. Among the highvolume centers represented by the authors, a range of 40100 patients are monitored by a single clinic nurse or APP with supervision from a physician. By following a treatment protocol and utilizing notifications to identify patients outside of threshold ranges, a large population of patients can be monitored without significant increase in staffing. While the hemodynamic management approach creates new roles and responsibilities, it simultaneously decreases time spent in traditional face-to-face encounters and/or telephone management.

\section{Knowledge Gaps and Future Directions}

A major unanswered question is whether HF management assisted by hemodynamic data affects mortality in addition to its known benefit in lowering HFH. Although CHAMPION demonstrated trends towards improved survival, it was not statistically powered to assess mortality. A prospective, randomized study (Hemodynamic-GUIDEd Management of Heart Failure, GUIDE-HF; Clinical trials NCT03387813) is underway to assess the impact of ambulatory PA pressure monitoring on long-term survival and hospitalization in chronic HF, coupled with quality of life and functional capacity. GUIDE-HF will enroll patients with a prior hospitalization or elevated NT-proBNP without a prior hospitalization and could extend the benefits of monitoring to those without a prior hospitalization. Communication directly to patients via a smart phone app may provide patients with their pressures directly as well as treatment recommendations.

The use of hemodynamic monitoring in other patient populations not included in CHAMPION is being actively explored. Ambulatory hemodynamic monitoring may facilitate early identification of stage D HF patients who should be referred for LVAD or heart transplantation [44]. After LVAD implantation, some patients continue to have significant functional limitations and/or abnormal hemodynamic profiles that can be improved with more intensive medical treatment or changes in device speed [45]. Case series have shown the utility of CardioMEMS in continuous flow LVAD populations [46-48]. A larger scale, multi-center study of the use of hemodynamic monitoring to optimize hemodynamics in LVAD patients (Clinical trials NCT03247829) is currently underway. Similarly, CardioMEMS provides a platform for investigating the hemodynamic effects of novel pharmacologic and device therapies, including SGLT2 inhibitors (Clinical Trials NCT03030222), MitraClip, [49], and therapies for pulmonary arterial hypertension.

\section{Conclusions}

An implantable wireless hemodynamic sensor is a novel technology that has shown significant promise in the treatment of chronic HF. Clinical management of PA pressures entails a shift in the management approach compared to conventional HF management guided by signs and symptoms alone. Further refinement of patient selection, workflows, and treatment algorithms are needed to improve outcomes in this growing patient population.

\section{Compliance with Ethical Standards}

Conflict of Interest Jacob Abraham has received grants from Abbott and speaker honorarium from Abbott.

Patrick McCann has received grants from Abbott and speaker honorarium from Novartis.

Maya Guglin has received research grants from Abbott.

Arvind Bhimaraj has received grants from Abbott and speaker honorarium from Abbott.

Terri Benjamin has received research grants from Abbott.

Monique Robinson has received research grants from Abbott.

Orvan Jonsson has received research grants from Abbott and speaker honoraria from Abbott and Novartis.

Scott Feitell has received research grants from Abbott and Johnson \& Johnson and speaker honoraria from Novartis and Johnson \& Johnson.

Kunjan Bhatt has received research grants from Abbott and speaker honoraria from Novartis and Pfizer.

Mosi Bennett has received research grants from Abbott and speaker honorarium from Abbott.

Tom Heywood has received research grants from Abbott and speaker honoraria from Abbott and Novartis.

Human and Animal Rights and Informed Consent All reported studies/ experiments with human or animal subjects performed by the authors have been previously published and complied with all applicable ethical standards (including the Helsinki declaration and its amendments, institutional/national research committee standards, and international/nation/institutional guidelines).

Open Access This article is licensed under a Creative Commons Attribution 4.0 International License, which permits use, sharing, adaptation, distribution and reproduction in any medium or format, as long as you give appropriate credit to the original author(s) and the source, provide a link to the Creative Commons licence, and indicate if changes were made. The images or other third party material in this article are included in the article's Creative Commons licence, unless indicated otherwise in a credit line to the material. If material is not included in the article's Creative Commons licence and your intended use is not permitted by statutory regulation or exceeds the permitted use, you will need to obtain permission directly from the copyright holder. To view a copy of this licence, visit http://creativecommons.org/licenses/by/4.0/. 


\section{References}

Papers of particular interest, published recently, have been highlighted as:

- Of importance

•- Of major importance

1. Abraham WT, Adamson PB, Bourge RC, Aaron MF, Costanzo MR, Stevenson LW, et al. Wireless pulmonary artery haemodynamic monitoring in chronic heart failure: a randomised controlled trial. Lancet. 2011;377:658-66.

2. Givertz MM, Stevenson LW, Costanzo MR, Bourge RC, Bauman JG, Ginn G, et al. Pulmonary artery pressure-guided management of patients with heart failure and reduced ejection fraction. J Am Coll Cardiol. 2017;70:1875-86.

3. Chaudhry SI, Mattera JA, Curtis JP, Spertus JA, Herrin J, Lin Z, et al. Telemonitoring in patients with heart failure. N Engl J Med. 2010;363:2301-9.

4. Ong MK, Romano PS, Edgington S, Aronow HU, Auerbach AD, Black JT, et al. Effectiveness of remote patient monitoring after discharge of hospitalized patients with heart failure: the better effectiveness after transition - heart failure (BEAT-HF) randomized clinical trial. JAMA Intern Med. 2016;176:310-8.

5. van Veldhuisen DJ, Braunschweig F, Conraads V, Ford I, Cowie $\mathrm{MR}$, Jondeau G, et al. Intrathoracic impedance monitoring, audible patient alerts, and outcome in patients with heart failure. Circulation. 2011;124:1719-26.

6. Felker GM, Anstrom KJ, Adams KF, Ezekowitz JA, Fiuzat M, Houston-Miller N, et al. Effect of natriuretic peptide-guided therapy on hospitalization or cardiovascular mortality in high-risk patients with heart failure and reduced ejection fraction: a randomized clinical trial. JAMA. 2017;318:713-20.

7. Stevenson LW. Tailored therapy to hemodynamic goals for advanced heart failure. Eur J Heart Fail. 1999;1:251-7.

8. Reddy YNV, El-Sabbagh A, Nishimura RA. Comparing pulmonary arterial wedge pressure and left ventricular end diastolic pressure for assessment of left-sided filling pressures. JAMA Cardiol. 2018;3:453-4.

9. Ghio S, Gavazzi A, Campana C, Inserra C, Klersy C, Sebastiani R, et al. Independent and additive prognostic value of right ventricular systolic function and pulmonary artery pressure in patients with chronic heart failure. J Am Coll Cardiol. 2001;37:183-8.

10. Lam CS, Roger VL, Rodeheffer RJ, Borlaug BA, Enders FT, Redfield MM. Pulmonary hypertension in heart failure with preserved ejection fraction: a community-based study. J Am Coll Cardiol. 2009;53:1119-26.

11. Tampakakis E, Shah SJ, Borlaug BA, Leary PJ, Patel HH, Miller WL, et al. Pulmonary effective arterial elastance as a measure of right ventricular afterload and its prognostic value in pulmonary hypertension due to left heart disease. Circ Heart Fail. 2018;11: e004436.

12. Chaudhry MA, Johnson A, Heywood JT. Stiff left atrial syndrome; prospects and possibilities. Retrospective analysis and review of the literature. Curr Hypertens Rev. 2019;15:17-21.

13. Vachiery JL, Adir Y, Barbera JA, Champion H, Coghlan JG, Cottin $\mathrm{V}$, et al. Pulmonary hypertension due to left heart diseases. J Am Coll Cardiol. 2013;62:D100-8.

14. Kjaergaard J, Akkan D, Iversen KK, Kober L, Torp-Pedersen C, Hassager C. Right ventricular dysfunction as an independent predictor of short- and long-term mortality in patients with heart failure. Eur J Heart Fail. 2007;9:610-6.

15. Costanzo MR, Stevenson LW, Adamson PB, Desai AS, Heywood JT, Bourge RC, et al. Interventions linked to decreased heart failure hospitalizations during ambulatory pulmonary artery pressure monitoring. JACC Heart Fail. 2016;4:333-44.

16.• Heywood JT, Jermyn R, Shavelle D, Abraham WT, Bhimaraj A, Bhatt $\mathrm{K}$, et al. Impact of practice-based management of pulmonary artery pressures in 2000 patients implanted with the Cardiomems sensor. Circulation. 2017;135:1509-17 Heywood et al. demonstrate results of a post-approval study of CardioMems. In this real-world setting, patients consistently transmitted pressure information. Patients in this study had higher baseline pulmonary artery pressure and experienced greater pressure reductions over time compared to those in the randomized pivotal trial.

17.• Desai AS, Bhimaraj A, Bharmi R, Jermyn R, Bhatt K, Shavelle D, et al. Ambulatory hemodynamic monitoring reduces heart failure hospitalizations in "real-world" clinical practice. J Am Coll Cardiol. 2017;69:2357-65 Desai et al show in a retrospective study using Medicare claims that implantation of a CardioMEMS sensor is associated with lower hospitalizations for HF and HF costs at six months following sensor implant.

18. Abraham J, Bharmi R, Jonsson O, Oliveira GH, Artis A, Valika A, et al. Association of ambulatory hemodynamic monitoring of heart failure with clinical outcomes in a concurrent matched cohort analysis. JAMA Cardiol. 2019;4:556-63.

19. Vaduganathan M, DeFilippis EM, Fonarow GC, Butler J, Mehra MR. Postmarketing adverse events related to the cardiomems hf system. JAMA Cardiol. 2017;2:1277-9.

20. Abraham J, Wang L, Lewis R, Callis K, Remick J, Spinelli KJ. Hemodynamic, therapeutic, and clinical outcomes of ambulatory pressure-guided heart failure management. VAD J. 2019;5:1 The authors show that a nurse-implemented treatment algorithm for CardioMEMS is safe and effective in a small, single-center analysis.

21. Naeije R, Gerges M, Vachiery JL, Caravita S, Gerges C, Lang IM. Hemodynamic phenotyping of pulmonary hypertension in left heart failure. Circ Heart Fail. 2017;10.

22. De Bruyne LK. Mechanisms and management of diuretic resistance in congestive heart failure. Postgrad Med J. 2003;79:268-71.

23. Rao VS, Planavsky N, Hanberg JS, Ahmad T, Brisco-Bacik MA, Wilson FP, et al. Compensatory distal reabsorption drives diuretic resistance in human heart failure. J Am Soc Nephrol. 2017;28: 3414-24.

24. Cook JA, Smith DE, Cornish LA, Tankanow RM, Nicklas JM, Hyneck ML. Kinetics, dynamics, and bioavailability of bumetanide in healthy subjects and patients with congestive heart failure. Clin Pharmacol Ther. 1988;44:487-500.

25. Vargo DL, Kramer WG, Black PK, Smith WB, Serpas T, Brater DC. Bioavailability, pharmacokinetics, and pharmacodynamics of torsemide and furosemide in patients with congestive heart failure. Clin Pharmacol Ther. 1995;57:601-9.

26. Brisco-Bacik MA, Ter Maaten JM, Houser SR, Vedage NA, Rao $\mathrm{V}$, Ahmad T, et al. Outcomes associated with a strategy of adjuvant metolazone or high-dose loop diuretics in acute decompensated heart failure: a propensity analysis. J Am Heart Assoc. 2018;7: e009149.

27. Buckley LF, Carter DM, Matta L, Cheng JW, Stevens C, Belenkiy $\mathrm{RM}$, et al. Intravenous diuretic therapy for the management of heart failure and volume overload in a multidisciplinary outpatient unit. JACC Heart failure. 2016;4:1-8.

28. Guglin M, Rivero A, Matar F, Garcia M. Renal dysfunction in heart failure is due to congestion but not low output. Clin Cardiol. 2011;34:113-6.

29. Hanberg JS, Sury K, Wilson FP, Brisco MA, Ahmad T, Ter Maaten $\mathrm{JM}$, et al. Reduced cardiac index is not the dominant driver of renal dysfunction in heart failure. J Am Coll Cardiol. 2016;67:2199-208.

30. Ahmad T, Jackson K, Rao VS, Tang WHW, Brisco-Bacik MA, $\mathrm{Chen} \mathrm{HH}$, et al. Worsening renal function in patients with acute 
heart failure undergoing aggressive diuresis is not associated with tubular injury. Circulation. 2018;137:2016-28.

31. Brisco MA, Zile MR, Hanberg JS, Wilson FP, Parikh CR, Coca $\mathrm{SG}$, et al. Relevance of changes in serum creatinine during a heart failure trial of decongestive strategies: insights from the dose trial. J Card Fail. 2016;22:753-60.

32. Steimle AE, Stevenson LW, Chelimsky-Fallick C, Fonarow GC, Hamilton MA, Moriguchi JD, et al. Sustained hemodynamic efficacy of therapy tailored to reduce filling pressures in survivors with advanced heart failure. Circulation. 1997;96:1165-72.

33. McMurray JJ, Packer M, Desai AS, Gong J, Lefkowitz MP, Rizkala AR, et al. Angiotensin-neprilysin inhibition versus enalapril in heart failure. N Engl J Med. 2014;371:993-1004.

34. Metkus TS, Mullin CJ, Grandin EW, Rame JE, Tampakakis E, Hsu $\mathrm{S}$, et al. Heart rate dependence of the pulmonary resistance $\mathrm{x}$ compliance (rc) time and impact on right ventricular load. PLoS One. 2016;11:e0166463.

35. Swedberg K, Komajda M, Bohm M, Borer JS, Ford I, DubostBrama A, et al. Ivabradine and outcomes in chronic heart failure (shift): a randomised placebo-controlled study. Lancet. 2010;376: 875-85.

36. Yancy CW, Jessup M, Bozkurt B, Butler J, Casey DE Jr, Drazner $\mathrm{MH}$, et al. 2013 accf/aha guideline for the management of heart failure: a report of the American college of cardiology foundation/ american heart association task force on practice guidelines. J Am Coll Cardiol. 2013;62:e147-239.

37. Zile MR, Baicu CF, Gaasch WH. Diastolic heart failureabnormalities in active relaxation and passive stiffness of the left ventricle. N Engl J Med. 2004;350:1953-9.

38. Adamson PB, Abraham WT, Bourge RC, Costanzo MR, Hasan A, Yadav $\mathrm{C}$, et al. Wireless pulmonary artery pressure monitoring guides management to reduce decompensation in heart failure with preserved ejection fraction. Circ Heart Fail. 2014;7:935-44.

39. Kapelios CJ, Bonou M, Vogiatzi P, Tzanis G, Mantzouratou P, Lund LH, et al. Association between high-dose spironolactone and decongestion in patients with acute heart failure: an observational retrospective study. Am J Cardiovasc Drugs. 2018;18:41522.

40. Butler J, Anstrom KJ, Felker GM, Givertz MM, Kalogeropoulos AP, Konstam MA, et al. Efficacy and safety of spironolactone in acute heart failure: the athena-hf randomized clinical trial. JAMA Cardiol. 2017;2:950-8.

41. Pitt B, Pfeffer MA, Assmann SF, Boineau R, Anand IS, Claggett B, et al. Spironolactone for heart failure with preserved ejection fraction. N Engl J Med. 2014;370:1383-92.

42. Yancy CW, Jessup M, Bozkurt B, Butler J, Casey DE Jr, Colvin MM, et al. $2017 \mathrm{acc} / \mathrm{aha} / \mathrm{hfsa}$ focused update of the $2013 \mathrm{accf} / \mathrm{aha}$ guideline for the management of heart failure: a report of the American college of cardiology/american heart association task force on clinical practice guidelines and the heart failure society of america. J Am Coll Cardiol. 2017;70:776-803.

43. Redfield MM, Anstrom KJ, Levine JA, Koepp GA, Borlaug BA, $\mathrm{Chen} \mathrm{HH}$, et al. Isosorbide mononitrate in heart failure with preserved ejection fraction. N Engl J Med. 2015;373:2314-24.

44. Kilic A, Katz JN, Joseph SM, Brisco-Bacik MA, Uriel N, Lima B, et al. Changes in pulmonary artery pressure before and after left ventricular assist device implantation in patients utilizing remote haemodynamic monitoring. ESC Heart Fail. 2019;6:138-45.

45. Uriel N, Sayer G, Addetia K, Fedson S, Kim GH, Rodgers D, et al. Hemodynamic ramp tests in patients with left ventricular assist devices. JACC Heart Fail. 2016;4:208-17.

46. Feldman DS, Moazami N, Adamson PB, Vierecke J, Raval N, Shreenivas $\mathrm{S}$, et al. The utility of a wireless implantable hemodynamic monitoring system in patients requiring mechanical circulatory support. ASAIO J. 2018;64:301-8.

47. George B, Hart A, Branam S, Guglin M. The utility of remote hemodynamic monitoring devices in patients with a ventricular assist device. VAD J. 2016;2:11.

48. Guglin M, George B, Branam S, Hart A. Cardiomems ${ }^{\mathrm{TM}}$ in lvad patients: a case series. VAD J. 2016;2:21.

49. Herrmann E, Ecke A, Eissing N, Fichtlscherer S, Zeiher AM, Assmus B. Daily non-invasive haemodynamic telemonitoring for efficacy evaluation of mitraclip(r) implantation in patients with advanced systolic heart failure. ESC Heart Fail. 2018;5:780-7.

Publisher's Note Springer Nature remains neutral with regard to jurisdictional claims in published maps and institutional affiliations.

\section{Affiliations}

\section{Jacob Abraham ${ }^{1} \cdot$ Patrick J. McCann ${ }^{2} \cdot$ Maya E. Guglin ${ }^{3}$ - Arvind Bhimaraj ${ }^{4} \cdot$ Terrie-Ann S. Benjamin $^{5}$. Monique R. Robinson ${ }^{6} \cdot$ Orvar T. Jonsson $^{7} \cdot$ Scott C. Feitell ${ }^{8} \cdot$ Kunjan A. Bhatt $^{9} \cdot$ Mosi K. Bennett $^{10} \cdot$ J.T. Heywood ${ }^{11} \cdot$ on $^{2}$ behalf of Hemodynamic Frontiers in Heart Failure (HF2) Investigators}

1 Center for Cardiovascular Analytics, Research and Data Science (CARDS), Providence Heart Institute, Providence St. Joseph Health, 9427 SW Barnes Road, Suite 594, Portland, OR 97225, USA

2 Palmetto Health University of South Carolina Medical Group, Columbia, SC, USA

3 Krannert Institute of Cardiology, Indiana University School of Medicine, Indianapolis, IN 46202, USA

4 Houston Methodist Debakey Heart and Vascular Institute and Methodist J.C. Walter Jr. Transplant Center, Houston, TX 77030, USA
5 Fairview Health Services and Medical Center, Houston, TX 77030 , USA

6 Advanced Heart Failure and Transplantation Center, Harrington Heart and Vascular Institute, University Hospitals Cleveland Medical Center, Cleveland, OH 44106, USA

7 Sanford Cardiovascular Institute, Sanford University of South Dakota School of Medicine, Vermillion, SD 57069, USA

8 Sands Constellation Heart Institute, University of Rochester Medical Center, Rochester, NY 14642, USA

9 Austin Heart Hospital, Austin, TX 78756, USA 